\title{
AN EQUATION FOR THE ISOTHERMS OF PURE SUB- STANCES AT THEIR CRITICAL TEMPERATURES
}

\author{
By Cyril H. Meyers
}

\begin{abstract}
$A n^{\prime \prime}$ empirical "equation" is presented for data"along the isotherms of pure substances at their critical temperatures and densities up to 1.1 times the critical density. The equation has five constants in addition to the universal gas constant. Of these five, one is limited to a single value, if a simple solution to the equation is to be obtained; three are determined by the critical conditions; and the remaining constant is determined empirically from data other than those for the critical state. Three of the five constants are independent of the substance, one is an explicit function of $R T_{c} / p_{c} V_{c}$, whereas the reciprocal of the fifth is found to be practically a linear function of $R T_{c} / p_{c} V_{c}$ for values of that ratio up to 3.8 or 3.9. This relation correlates the isotherms for various substances, so that data on two well chosen substances, such as hydrogen and carbon dioxide, suffice to determine the fifth constant and consequently the critical isotherm for other substances, for which $R T_{c} / p_{c} V_{c}$ is not greater than 3.8. For substances such as ammonia (for which $R T_{c} / p_{c} V_{c}$ is 4.08 ), the fifth constant may not conform to the linear relation.

The equation represents the data for 12 of 13 substances within the experimental error, the exceptional substance being water (for which $R T_{c} / p_{c} V_{c}$ is 4.34 ).

At low densities the equation reduces to a simpler form which expresses $p V / R T$ as a quadratic function of density.

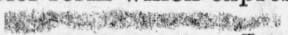

The fugacity along the critical isotherm can be calculated from the equation. The fugacity at the critical state is approximately two-thirds the critical pressure for all substances. The fungacity of $\mathrm{CO}_{2}$ is calculated at 1 atmosphere and at the critical pressure.
\end{abstract}

\section{CONTENTS}

I. Introduction Page

II. Failure of the law of corresponding states.

III. Equation for the isotherm at the critical temperature

IV. Representation of the experimental data

1. Hydrogen

2. Oxygen and nitrogen

3. Methane

4. Ethane

5. Propane

6. Carbon dioxide.

7. Propylene

8. Normal butane.

9. Ethyl ether

10. Normal heptane

11. Ammonia

12. Water

V. Virial coefficients for the isotherm at the critical temperature

VI. Fugacity along the isotherms at the critical temperature

VII. Summary 


\section{INTRODUCTION}

The isotherm at the critical temperature forms a convenient base from which to proceed in tabulating the properties of vapors. Consequently, a mathematical representation of the critical isotherm is desirable. A mathematical representation of the critical isotherm is especially difficult, since at the critical temperature not only must the equation represent the experimental data, but it must satisfy the conditions that the first and second derivatives of pressure with respect to volume (or density) must equal zero at the critical state.

The notation used in this paper is as follows: $R$, the universal gas constant; $T$, absolute temperature; $p$, absolute pressure; $V$, molal

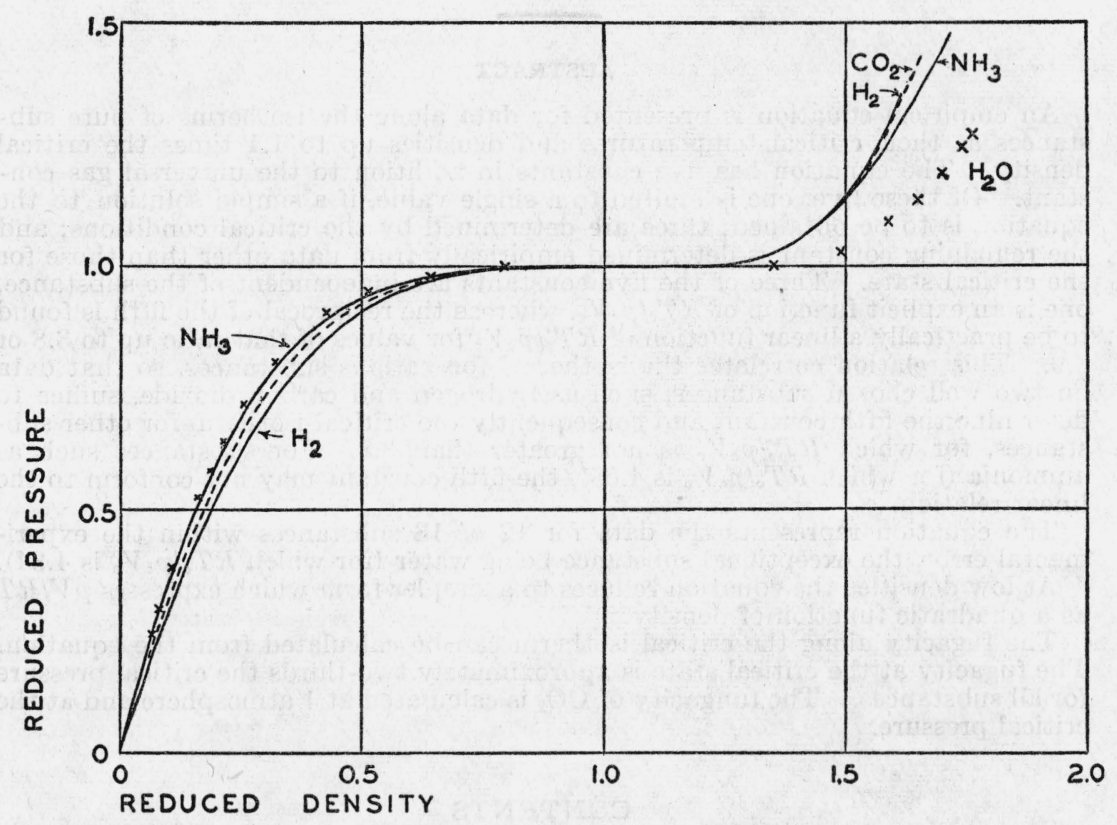

FIgure 1.-Comparison of reduced pressures at corresponding densities along the critical isotherms of four substances.

volume; $c$, subscript representing critical state; $X, V_{c} / V$ or reduced density; $f$, fugacity; $m$, number of a term in a series; $n$, empirical constant; and $K$, empirical constant.

\section{FAILURE OF THE LAW OF CORRESPONDING STATES}

The law of corresponding states has been used occasionally for predicting the properties of pure substances from the properties of other substances, but this procedure is subject to rather large errors. According to the law of corresponding states, the data for the critical isotherms of all substances would lie on a single curve on a graph with reduced pressure as ordinate and reduced density (or volume) as abscissa. Such a graph, which is illustrated in figure 1 for $\mathrm{H}_{2}, \mathrm{CO}_{2}$, $\mathrm{NH}_{3}$, and $\mathrm{H}_{2} \mathrm{O}$, indicates that the assumption of the law of corresponding states may lead to errors as large as 10 percent of the pressure. 
For densities less than the critical density, the position of the curves appears to be related to the quantity $R T_{c} / p_{c} V_{c}$. The greater the value of $R T_{c} / p_{c} V_{c}$, the greater is the reduced pressure corresponding to any given reduced density, $X$. This has been found to be true also for other substances studied but is omitted from figure 1 to avoid confusion.

The arrangement for densities greater than the critical is in general reversed from that at smaller densities and appears not to be entirely a function of $R T_{c} / p_{c} V_{c}$, although this irregularity may be due in part to erroneous critical densities. The data for water are so conspicuously

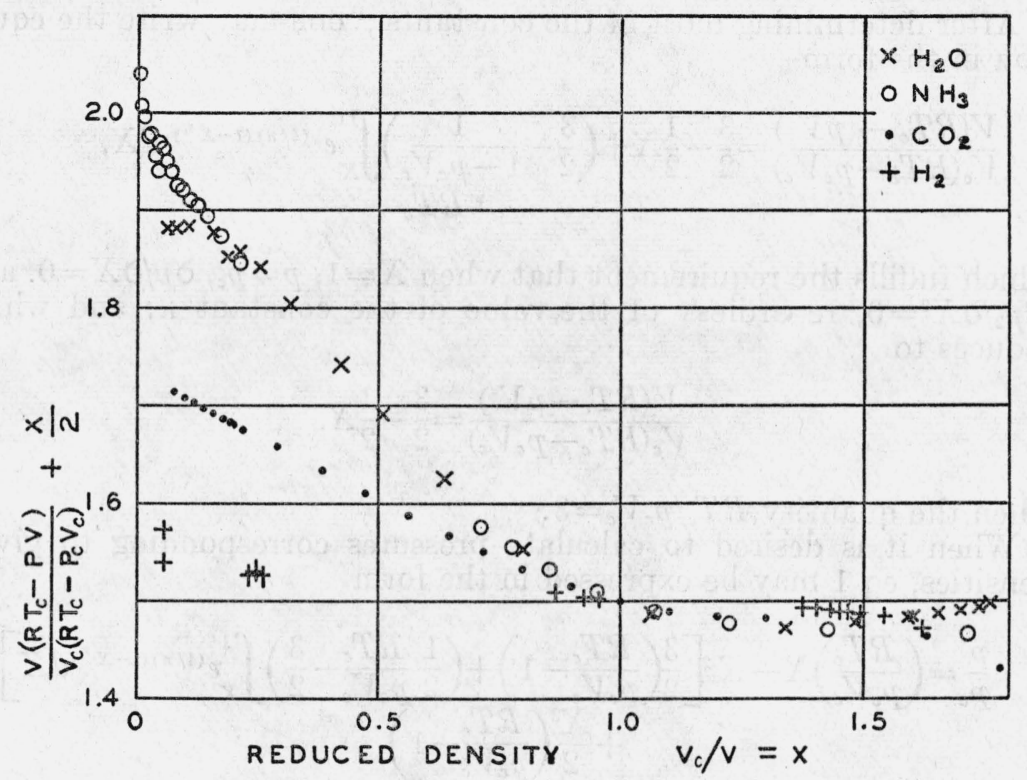

FIGURE 2.-Comparison of data for the critical isotherms of four substances.

far below the other curves in figure 1 that the irregularity appears to be greater than any experimental error possible in the critical density.

\section{EQUATION FOR THE ISOTHERM AT THE CRITICAL TEMPERATURE}

The differences between the critical isotherms of different substances at densities less than the critical are better illustrated by plotting the quantity

$$
\frac{V\left(R T_{c}-p V\right)}{V_{c}\left(R T_{c}-p_{c} V_{c}\right)}+\frac{X}{2}
$$

as ordinate, and the reduced density, $X$, as abscissa. Such a graph, in which the same data as in figure 1 are represented, is illustrated in figure 2. The data are on smooth curves which deviate more or less from the straight line,

$$
\frac{V\left(R T_{c}-p V\right)}{V_{c}\left(R T_{c}-p_{c} V_{c}\right)}=\frac{3}{2}-\frac{1}{2} X
$$


Numerous attempts to find directly a function to represent the curves in figure 2 have failed-the functions always yielded graphs with too much curvature at low densities.

When the slopes of the curve for $\mathrm{CO}_{2}$ in figure 2 were plotted against density, the resulting graph was remarkably similar to that for the function $y=a e^{-b x^{n}}$. This suggested the general equation

$$
\frac{V\left(R T_{c}-p V\right)}{V_{c}\left(R T_{c}-p_{c} V_{c}\right)}=L-M X+a \int_{X}^{1} e^{-b X^{n}} d X
$$

After determining most of the constants,${ }^{1}$ one may write the equation in the form

$$
\frac{V\left(R T_{c}-p V\right)}{V_{c}\left(R T_{c}-p_{c} V_{c}\right)}=\frac{3}{2}-\frac{1}{2} X+\left(\frac{3}{2}-\frac{1}{1-\frac{p_{c} V_{c}}{R T_{c}}}\right) \int_{X}^{1} e^{(4 / n)\left(1-X^{n}\right)} d X
$$

which fulfills the requirement that when $X=1, p=p_{c}, \partial p / \partial X=0$, and $\partial^{2} p_{2} / \partial X^{2}=0$, regardless of the value of the constant $n$; and which reduces to

$$
\frac{V\left(R T_{c}-p V\right)}{V_{c}\left(R T_{c}-p_{c} V_{c}\right)}=\frac{3}{2}-\frac{1}{2} X
$$

when the quantity $R T_{c} / p_{c} V_{c}=3$.

When it is desired to calculate pressures corresponding to given densities, eq 1 may be expressed in the form

$$
\begin{gathered}
\frac{p}{p_{c}}=\left(\frac{R T_{c}}{p_{c} V_{c}}\right) X-X^{2}\left[\frac{3}{2}\left(\frac{R T_{c}}{p_{c} V_{c}}-1\right)+\left(\frac{1}{2} \frac{R T_{c}}{p_{c} V_{c}}-\frac{3}{2}\right) \int_{X}^{1} e^{(4 / n)\left(1-X^{n}\right)} d X\right] \\
+\frac{X^{3}}{2}\left(\frac{R T_{c}}{p_{c} V_{c}}-1\right)
\end{gathered}
$$

For use in calculations with the equation in either form, eq 1 or 3 , values of $\int_{X}^{1} e^{(4 / n)\left(1-X^{n}\right)} d X$ are given in table 1 for various values of $1 / n$ and $X$. For data of moderate accuracy, for example, pressures accurate to a few parts in a thousand, graphic methods of interpola-

1 The procedure is as follows: For brevity let $F$ represent temporarily the fraction $R T_{c} / p_{\circ} V_{c}$. Then the solution of the general equation for $p / p_{c}$ gives

$$
p / p_{c}=F X-L X^{2}(F-1)+M X^{3}(F-1)-a X^{2}(F-1) \int_{x}^{1} e^{-b X^{n}} d X .
$$

By differentiation

and

$$
\frac{\delta p / p_{0}}{\delta X}=F-2 L X(F-1)+3 M X^{2}(F-1)-2 a X(F-1) \int_{X}^{1} e^{-b X^{n}} d X+a X^{2}(F-1) e^{-b X^{n}},
$$

$$
\frac{\partial^{2} p / p_{0}}{\partial X^{2}}=-2 L(F-1)+6 M X(F-1)-2 a(F-1) \int_{x}^{1} e^{-b x^{n}} d X+4 a X(F-1) e^{-b x^{n}}-a b n X^{n+1}(F-1) e^{-b x^{n}}
$$

By substitution of the critical conditions $p / p_{c}=1, X=1, \partial\left(p / p_{c}\right) / \partial X=0$, and $\partial^{2}\left(p / p_{c}\right) / \partial X^{2}=0$ in these equations, one obtains three simultaneous equations, from which some of the constants can be eliminated.
The solution is

$$
b n=\frac{6-4 F /(F-1)}{2-M-F /(F-1)}
$$

Apparently $M$ can have only one value (one-half) if a simple solution is to be obtained. Assumption of this value leads to values for the other constants as written in eq.1 
tion are satisfactory, but for greater accuracy, it may be better to calculate directly the values of the integral ${ }^{2}$ for the chosen value of $n$ and the desired values of $X$. For values of $X$ up to 1.1 or 1.2 , the integral is easily calculated by expanding $e^{-(4 / n) X^{n}}$ into series form and integrating each significant term of the series. For greater values of $X$ this method becomes increasingly laborious, and numerical integration of the quantity $e^{-(4 / n) X^{n}}$ becomes the easier method. Where data are to be calculated for a large number of values of $X$ between 0.8 and 1.2 , it is easier to calculate only a few values of pressure in this range and to obtain the remainder by interpolation between these pressures.

To maintain the correct relations at the critical point, the quantity $R T_{c} / p_{c} V_{c}$ should be treated consistently as an exact number in calculating the coefficients of the various terms in eq 3 , and it is advisable in calculating pressures to carry each term of the equation to more decimal places than the accuracy of the critical data would warrant.

TABLE 1.-Values of $\int_{X}^{1} e^{(4 / n)\left(1-X^{n}\right)} d X$ for various values of $X$ and $1 / n$

\begin{tabular}{|c|c|c|c|c|c|c|c|}
\hline \multirow{2}{*}{$x$} & \multicolumn{7}{|c|}{ Values of $1 / n$} \\
\hline & 0 & 0.05 & 0.1 & 0.15 & 0.2 & 0.25 & $1 / 3$ \\
\hline 0 & & 1. 2104 & 1. 4428 & 1. 6997 & 1. 9833 & 2. 2965 & 2.8924 \\
\hline & .9 & 1.0882 & 1. 2936 & 1. 5175 & 1.7607 & 2. 0247 & 2.5132 \\
\hline 2 & .8 & .9661 & 1. 1444 & 1. 3353 & 1. 5382 & 1. 7530 & 2. 1357 \\
\hline .3 & .7 & .8439 & .9952 & 1. 1531 & 1.3158 & 1. 4824 & 1.7645 \\
\hline .4 & .6 & .7218 & .8460 & .9709 & 1. 0943 & 1. 2147 & 1.4065 \\
\hline .5 & .5 & .5997 & .6969 & .7893 & .8751 & .9541 & 1. 0710 \\
\hline .6 & .4 & .4775 & .5479 & . 6092 & .6616 & .7063 & .7675 \\
\hline .7 & .3 & $\begin{array}{r}.3554 \\
2333\end{array}$ & $\begin{array}{l}.3996 \\
2539\end{array}$ & .4333 & .4591 & .4793 & .5046 \\
\hline & .2 & $\begin{array}{l}.2333 \\
.1124\end{array}$ & $\begin{array}{l}.2539 \\
.1166\end{array}$ & $\begin{array}{r}.2668 \\
.1186\end{array}$ & $\begin{array}{l}.2754 \\
.1197\end{array}$ & $\begin{array}{l}.2815 \\
.1204\end{array}$ & $\begin{array}{l}.2878 \\
.1212\end{array}$ \\
\hline 1. 0 & $0^{-2}$ & 0 & 0 & 0 & 0 & & 0 \\
\hline 1.1 & 0 & -.0696 & -.0773 & -.0794 & -.0803 & -.0809 & -.0814 \\
\hline & 0 & -.0781 & -.1080 & -.1193 & -.1253 & -.1286 & -.1317 \\
\hline $\begin{array}{l}1.3 \\
1.4\end{array}$ & $\begin{array}{l}0 \\
0\end{array}$ & $\begin{array}{l}=.0781 \\
-.0781\end{array}$ & $\begin{array}{l}=.1124 \\
-.1127\end{array}$ & $\begin{array}{l}-.1328 \\
-.1353\end{array}$ & $\begin{array}{l}-.1452 \\
-.1517\end{array}$ & $\begin{array}{l}-.1526 \\
-.1627\end{array}$ & $\begin{array}{l}=.1601 \\
-.1747\end{array}$ \\
\hline 1.5 & 0 & -.0781 & -.1128 & -.1358 & -.1532 & -.1662 & -.1813 \\
\hline$\infty$ & & -.0781 & -.1128 & -.1359 & -.1534 & -.1673 & -.1855 \\
\hline
\end{tabular}

The constant $n$ is the only quantity in eq 1 which is not explicitly determined by the critical data. The most convenient method for

\footnotetext{
? It may be of academic interest to note that for $n=\infty$, when $X<1$,

and when $\boldsymbol{X}>1$,

$$
\int_{X}^{1} e^{(t / n)\left(1-x^{n}\right)} d X=1-X
$$

When $n=0$

$$
\int_{x}^{1} e(1 / n)\left(1-x^{n}\right) d X=0 .
$$$$
\int_{X}^{1} e^{(4 / n)\left(1-x^{n}\right)} d X=\int_{X}^{1} e^{-t \ln x} d X-\int_{X}^{1} \frac{d X}{X^{t}}=\frac{1}{3 X^{3}}-\frac{1}{3} .
$$

As an aid in checking calculations, it may be of interest to note that

$$
\int_{0}^{\infty} e^{-y^{n}} d y=(1 / n) \Gamma(1 / n) .
$$

See Bierens de Haan, Nouvelles Tables d'Intégrales Définies, 1867, table 81, formula 8. (Printed by J. C. Drabbe.) Formulas for calculating $(1 / n) \Gamma(1 / n)$ are given in Jahnke-Emde Funkionentafeln 3d (revised)
} edition, p. 10, published by G. B. Teubner, Leipzig and Berlin (1938). Then

$$
\int_{0}^{\infty} e^{(4 / n)\left(1-x^{n}\right)} d X=(n / 4)^{1 / n} e^{4 / n} \int_{0}^{\infty} e^{-s^{n}} d y=(n / 4)^{1 / n} e^{4 / n}(1 / n) \Gamma(1 / n) .
$$


determining $n$ is to substitute the critical data in the equation and to calculate the quantity

$$
\left(\frac{V\left(R T_{c}-p V\right)}{V_{c}\left(R T_{c}-p_{c} V_{c}\right)}-\frac{3}{2}+\frac{1}{2} X\right) /\left(\frac{3}{2}-\frac{1}{1-\left(p_{c} V_{c}\right) / R T_{c}}\right),
$$

which is equal to

$$
\int_{X}^{1} e^{(4 / n)\left(1-X^{n}\right)} d X
$$

The corresponding value of $n$ is obtained by interpolation in table 1 . For this purpose, experimental data at slightly less than one-half the critical density are most desirable, since calculations from eq 1 at various densities with the same critical data, but with two slightly different values of $n$, yield two sets of calculated pressures which differ by a maximum amount at a little less than one-half the critical density and which become equal as $X$ approaches 0 or 1 .

Of the three critical data, the critical volume is usually known with the least accuracy. Fortunately, however, there is a compensating action between the various terms of eq 1 , such that a change in the critical volume alone produces very little change in the pressures calculated at given densities.

A limit which the constant $n$ can neither equal nor exceed, if eq 1 is to represent properly the critical state, is afforded by consideration of the third and higher derivatives of pressure with respect to density.

The value at the critical state of the third and higher derivatives is probably beyond experimental determination. It is reasonable to suppose, however, that for densities less than the critical density, the pressure does not exceed the critical, and that for densities greater than the critical density no pressure less than the critical exists. This supposition leads to the conclusion that if all derivatives of pressure with respect to density up to and including any derivative of odd order are zero, then the derivative of next higher (even) order must also be zero.

One finds by differentiation of eq 1 for the condition $\mathrm{X}=1$, that $\partial^{3} p / \partial X^{3}$ is zero when

$$
n=\left(2 \frac{R T_{c}}{p_{c} V_{c}}-3\right) /\left(\frac{R T_{c}}{p_{c} V_{c}}-3\right)
$$

and that within the useful range of $n, \partial^{4} p / \partial X^{4}$ is zero only when $n$ is approximately 6.7. Hence one may say that in general $n$ can neither equal nor exceed the ratio

$$
\left(2 \frac{R T_{c}}{p_{c} V_{c}}-3\right) /\left(\frac{R T_{c}}{p_{c} V_{c}}-3\right)
$$

The fact that the values of the constant $n$ determined for various substances usually are less than

$$
\left(2 \frac{R T_{c}}{p_{c} V_{c}}-3\right) /\left(\frac{R T_{c}}{p_{c} V_{c}}-3\right)
$$

by considerable amounts does not preclude the possibility of $\partial^{3} p / \partial X^{3}$ (and $\partial^{4} p / \partial X^{4}$ ) actually being zero at the critical point. The equation 
is only empirical and therefore not exact. In the neighborhood of the critical point only very minute differences exist between the pressures calculated from the values of $n$ which were used and from those which equal

$$
\left(2 \frac{R T_{c}}{p_{c} V_{c}}-3\right) /\left(\frac{R T_{c}}{p_{c} V_{c}}-3\right) .
$$

For example, the reduced pressure of normal heptane calculated for $X=0.9$ and $n=3.66$ is 0.9997 , whereas the use of $n=5$ or approximately

$$
\left(2 \frac{R T_{c}}{p_{c} V_{c}}-3\right) /\left(\frac{R T_{c}}{p_{c} V_{c}}-3\right)
$$

gives 0.99997 for the reduced pressure - a difference of about 3 parts in 10,000 . The experimental data in this case indicate an intermediate value.

The method described for determining $n$ from experimental data was used for $\mathrm{H}_{2} \mathrm{O},\left(R T_{c} / p_{c} V_{c}=4.335, n=3.12\right) ; N H_{3}\left(R T_{c} / p_{c} V_{c}=\right.$ $4.075, n=3.20)$ and for $\mathrm{CO}_{2}\left(R T_{c} / p_{c} V_{c}=3.649, n=5.48\right)$. A value of $n$ for $\mathrm{H}_{2}\left(R T_{c} / p_{c} V_{c}=3.203, n=16.6\right)$ was chosen more or less arbitrarily as being equal to

$$
\left(2 \frac{R T_{c}}{p_{c} V_{c}}-3\right) /\left(\frac{R T_{c}}{p_{c} V_{c}}-3\right),
$$

since the experimental data were best represented by the use of as large a value of $n$ as possible. Although this procedure is mathematically incorrect, the errors introduced are practically negligible. As indicated in table 3, the calculated reduced pressure at $X=1.1$ is 0.9999 , that is low by 1 or more parts in 10,000 .

In figure 3 the value of $R T_{c} / p_{c} V_{c}$ is plotted as ordinate and that of $1 / n$ as abscissa. The dotted curve indicates the values of $1 / n$ for which $\partial^{3} p / \partial X^{3}=0$ at the critical state, whereas the values for the substances mentioned are indicated by the labeled crosses. The continuous, straight line in figure 3 through the point for $\mathrm{H}_{2}$ and that for $\mathrm{CO}_{2}$ passes between the points representing water and ammonia. The values of $n$ given in table 2 for other substances were obtained from this line and were found to represent the data within the experimental accuracy.

\section{REPRESENTATION OF THE EXPERIMENTAL DATA}

Although table 2 contains the critical temperatures, pressures, and volumes for the substances discussed in this paper, the main purpose of this table is to present the empirical constants actually used in representing the critical isotherms. For this reason some of the data are given to more places than is warranted by the experimental accuracy. As noted at the bottom of the table, data differing slightly from those chosen by the authors have been used in some instances. 


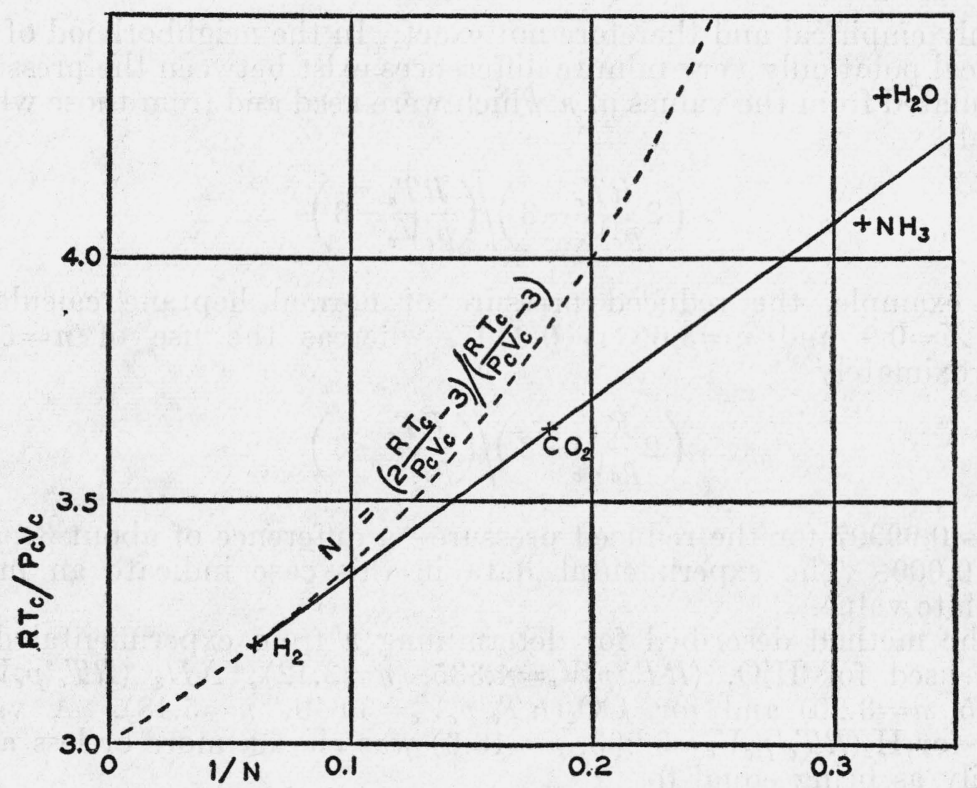

FigURe 3.-The value of $1 / n$ as a function of $R T_{c} / p_{c} V_{c}$.

TABLE 2.-Constants used in equations for the critical isotherms

\begin{tabular}{|c|c|c|c|c|c|c|c|}
\hline Substance & $T_{c^{\mathrm{a}}}$ & $P_{e}$ & $V_{0} b^{b}$ & $\frac{R T_{c}}{P_{c} V_{c}}$ & $\frac{P_{c} V_{c}}{R T_{c}}$ & $1 / n$ & $\begin{array}{l}\text { Refer- } \\
\text { ence }\end{array}$ \\
\hline Hydrogen. & $\begin{array}{l}{ }^{\circ} \mathrm{K} \\
33.18\end{array}$ & $\begin{array}{l}\text { atm } \\
(13.03)\end{array}$ & $0.002909 \mathrm{~A}$ & 3. 203 & 0.3122 & $0.060\}$ & 1 \\
\hline Oxygen & 154. 27 & 49.713 & $.003324 \mathrm{~A}$ & $\begin{array}{l}3.273 \\
\text { 3. } 422\end{array}$ & $\begin{array}{l}.3005 \\
.2922\end{array}$ & .080 & 1 \\
\hline Nitrogen.- & 125.96 & 33.490 & $.004027 \mathrm{~A}$ & 3. 422 & .2922 & .120 & 1 \\
\hline Methane_-. & 191.1 & 45.8 & .0993 & 3. 448 & .2901 & .128 & 2 \\
\hline Ethane & 305.43 & 48.20 & & 3. 513 & .2847 & .145 & 3 \\
\hline Propane & 369.96 & 42.004 & $(.201)$ & 3. 592 & .2784 & .167 & 4 \\
\hline $\mathrm{CO}_{2}$ & 304.197 & 72.885 & .004 & 3. 649 & .2740 & & 5 \\
\hline Propylene-... & 364.56 & 45.4 & .180 & 3. 660 & .2732 & .184 & 7 \\
\hline $\begin{array}{l}n \text {-Butane- } \\
\text { Ethyl ether. }\end{array}$ & 425.16 & 37.468 & $(.2544)$ & 3. 660 & .2732 & .184 & 8 \\
\hline$n$-Heptane... & 540.17 & 27.00 & .416 & $\begin{array}{l}\text { 5. } \\
\text { 3. } 946\end{array}$ & .2534 & .264 & $10^{y}$ \\
\hline Ammonis & & & & & & & \\
\hline & & & .0730 & 4. 075 & .2454 & .312 & \\
\hline Water. & 047.28 & 219.4 & .0558 & 4. 335 & .2368 & .32 & 15 \\
\hline
\end{tabular}

a The temperatures for $\mathrm{H}_{2}, \mathrm{O}_{2}$, and $\mathrm{N}_{2}$ are as reported in the reference cited. In other instances, ${ }^{\circ} \mathrm{K}=$ $273.16+{ }^{\circ} \mathrm{C}$.

b Liters per mole, unless marked "A", in which cases Amagat units were used. The numbers in parentheses are different from those given in the references cited. The reasons for these changes are explained in the text.

\section{REFERENCES}

1. F.P. G. A. J. van Agt, Comm. Phys. Lab. Univ. Leiden No. 176c, 16 (1925).

2. C. S. Cragoe, Int. Critical Tables 3, 230 (1928).

3. J. A. Beattie, C. Hadlock, and N. Poffenberger, J. Chem. Phys. 3, 93 (1935).

4. J. A. Beattie, N. Poffenberger, and C. Hadlock, J. Chem. Phys. 3, 96 (1935)

5. A. Michels, C. Michels, and H. Wouters, Proc. Roy. Soc. (London) [A] 153, 201 (1935).

6. C. H. Meyers and M. S. Van Dusen, BS J. Research 10, 381 (1933) RP538.

7. W. E. Vaughan and N. R. Graves, Ind. Eng. Chem. 32, $1252(1940)$.

8. J. A. Beattie, G. L. Simard, and Gouq-Jen Su, J. Am. Chem. Soc. 61, 24 (1939).

9. Erich Schroer, Z. physik. Chem. [A] 140, 241 (1929).

10. J. A. Beattie and W. C. Kay, J. Am. Chem. Soc. 59, 1586 (1937).

11. C. S. Cragoe, C. H. Meyers, and C. S. Taylor, BS Sci. Pap. 16, 1 (1920) S369.

12. F. G. Keyes, J. Am. Chem. Soc. 53, 965 (1931).

13. C. S. Cragoe and D. R. Harper, 3d, BS Sci. Pap. 17, 287 (1922) S420.

14. F. G. Keyes, L. B. Smith, and H. T. Gerry, Proc. Am. Acad. 70, 319 (1935).

15. N. S. Osborne, H. F. Stimson, and D. C. Ginnings, J. Research NBS 23, 261 (1939) RP1229. 
A knowledge of the weight of the normal liter is involved in the use of Amagat units, but this quantity is so much more accurately known than the other data involved that it is considered unnecessary to quote the source of the data used for the normal liter.

\section{HYDROGEN}

Since a discrepancy of about 2 percent exists between the experimental data for the superheated vapor of hydrogen and those for the saturated vapor, ${ }^{3}$ two sets of constants were used in eq 3 for calculating values of $p / p_{c}$. One set with $p_{c}=13.03 \mathrm{~atm}$ (see table 2) was used in calculating values used for comparing with the superheat data in figure 4 , and a second set was used with $p_{c}=12.75$ atm for comparison with tables which may be based upon this value of the critical pressure. The values calculated from these two sets of constants are given later in columns 2 and 3 , respectively, of table 3 . Although there is a considerable difference at low densities between the reduced pressures calculated from these two sets of constants, the absolute

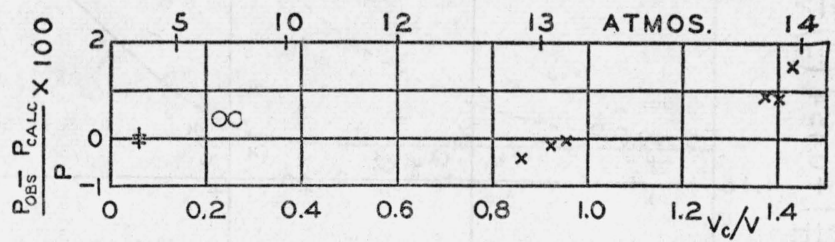

FIGURE 4.-Comparison of pressures calculated from equation 3 with pressures observed for hydrogen.

+ Interpolated from data by G. P. Nijhoff and W. H. Keesom in Comm. Phys. Lab. Univ. Leiden No. $188 \mathrm{e}, 17$ (1924-28).

$\mathrm{O}$ Averages of data by H. K. Onnes and P. M. Penning in Comm. Phys. Lab. Univ. Leiden No. 165b, $15(1920-26)$.

X C. A. Crommelin and J. C. Swallow, ?Comm. Phys. :Lab. Univ. ${ }_{n}^{*}$ Leiden No. 172a 16 (1924-26).

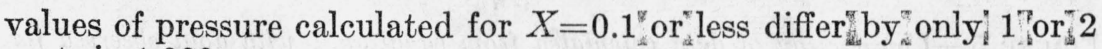
parts in 1,000 .

Figure 4 presents a comparison of experimental and calculated pressures for hydrogen. The agreement is as good as can be expected in view of the discrepancy already mentioned. The two plus marks at $X=0.058$ represent the largest and the smallest pressures which were obtained by different assumptions in interpolating between the experimental data at other temperatures. The trend of the crosses indicates that this isotherm is above the critical temperature. This fact, combined with the 2-percent discrepancy already mentioned, suggests a possible error in temperature measurement.

\section{OXYGEN AND NITROGEN}

The experimental data for both oxygen and nitrogen are at irregular intervals and therefore not subject to easy interpolation to the critical temperature. For the purpose of comparing these data, use is made of an equation developed by Leib, ${ }^{4}$ which, for diatomic gases, may be written in the form

$$
\frac{p V}{R T}=\frac{K\left(T_{c} / T\right)^{5 / 2} V_{c} / V}{e^{K\left(T_{c} / T /\right)^{5 / 2} V_{c} / V}-1}
$$

I H. K. Onnes, C. A. Crommelin, and P. G. Cath, Comm. Phys. Lab. Univ. Leiden No. 151c, 14 (1914-22).
E. F. Leib, Trans. Am. Soc. Mech. Engrs. 63, 157_(1941). 
and may be expanded into the form

$$
p V / R T=1-(K / 2)\left(T_{c} / T\right)^{5 / 2} V_{c} / V+\left(K\left(T_{c} / T\right)^{5 / 2} V_{c} / V\right)^{2} / 12-\ldots
$$

At low densities this leads to

$$
\left(T / T_{c}\right)^{5 / 2}\left(V / V_{c}\right)(1-p V / R T)+(K / 2)^{2}\left(T_{c} / T\right)^{5 / 2}\left(V_{c} / V\right) / 3=K / 2,
$$

where $K / 2$ is a single arbitrary constant which may be determined from the experimental data. Equation 1 may be expanded to a somewhat similar series, in which 1.157 corresponds to $K / 2$ and 0.454 to the third virial coefficient (see table 4). According to Leib, the third virial coefficient would then be one-third of $(K / 2)^{2}$, or 0.446 . With the choice of coordinates in figures 5 and 6 , the left-hand portion of the continuous curve representing eq 1 must be horizontal. The

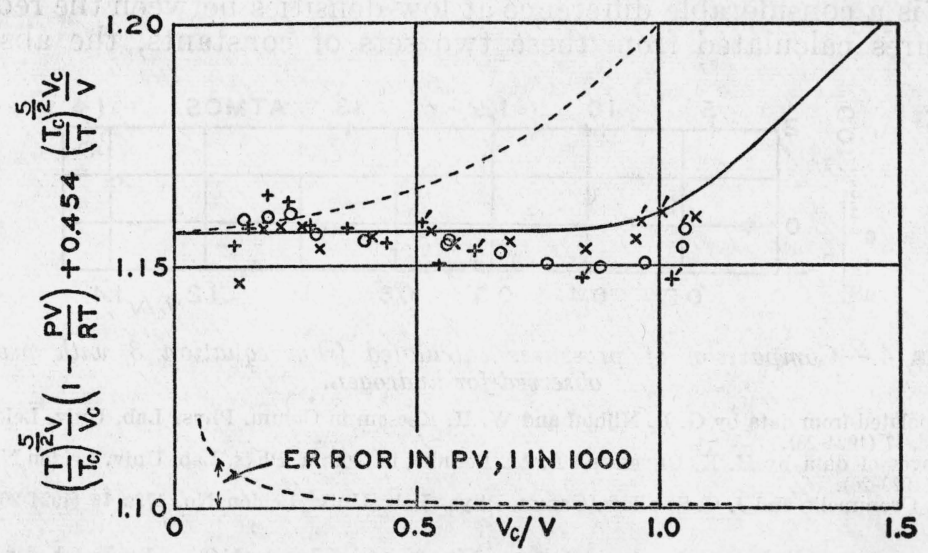

FIgURE 5.-Comparison of data calculated from equation 1 with data observed for oxygen.

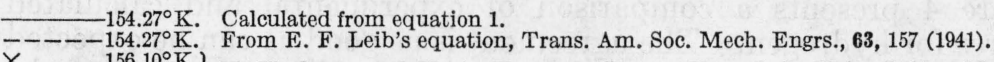
$\begin{array}{ll}\mathrm{X} & 156.10^{\circ} \mathrm{K} . \\ + & 157.08^{\circ} \mathrm{K} \\ + & 159.11^{\circ} \mathrm{K} .\end{array}$ H. K. Onnes and H. A. Kuypers, Comm. Phys. Lab. Univ. Leiden No. $169 \mathrm{a}, 16$

dotted curve just above the continuous curve represents Leib's equation. Leib's theory indicates that with the introduction of $\left(T_{c} / T\right)^{5} / 2$, the data for the different isotherms should lie on a single curve, which is approximately the case at the lower densities. Figure 5 indicates that eq 1 represents the data for oxygen within the precision of the experimental data. The data for nitrogen are more precise, and it would appear in figure 6 that at the higher densities, the critical isotherm calculated from eq 1 is slightly too low to correspond to the uniform spacing per degree of the different isotherms. This must be interpreted as a discrepancy between the reported critical data and the other isothermal data, rather than a failure of eq 1 , since the form of the calculated isotherm appears to be correct and, at the critical state, the equation is determined exactly by the critical data substituted in it.

The dotted curve at the bottom of figures 5 and 6 provides means for estimating the errors in pressure equivalent to deviations in the ordinate. 
All observations made on the oxygen, using a piezometer with a reported normal volume of $1820.6,{ }^{5}$ are designated in figure 5 with a prime mark. These points deviate systematically from smooth curves through the other data. The deviation corresponds in size to an

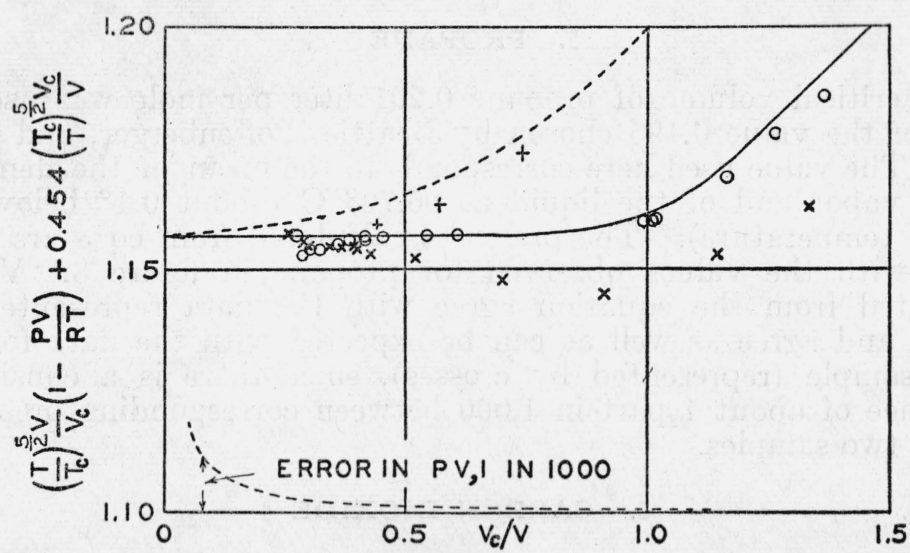

Figure 6.-Comparison of data calculated from equation 1 with data observed for nitrogen.

$-125.96^{\circ} \mathrm{K}$. Calculated from equation 1

$---125.96^{\circ} \mathrm{K}$. From E. F. Leib's equation, Trans. Am. Soc. Mech. Engrs., 63,157 (1941).

$\left.+\quad 124.51^{\circ} \mathrm{K}.\right\}$ H. K. Onnes and A. Th. van Urk, Comm. Phys. Lab. Univ. Leiden No. $169 \mathrm{~d}, 16$ × $\left.\quad 128.63^{\circ} \mathrm{K}.\right\} \quad(1924-26)$.

error of 1 gram in the weight of mercury in calibrating the small end of that particular piezometer.

\section{METHANE}

There are no data for methane at temperatures near the critical, but the calculated pressures are in agreement with those extrapolated from the observed data. ${ }^{6}$

\section{ETHANE}

A comparison of the pressures calculated from eq 3 with the observed data for ethane is given in figure 7 . The critical constants were those

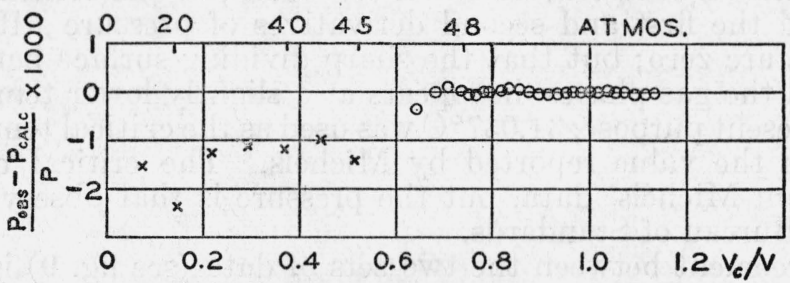

FIGURE 7.-Comparison of pressures calculated from equation 3 with pressures observed for ethane.

X J. A. Beattie, C. Hadlock, and N. Poffenberger, J. Chem. Phys. 3, 93; 1935.

O. A. Beattie, Gouq-Jen Su, and G. L. Simard, J. Am. Chem. Soc. 61, 924 (1939).

obtained from the data near the critical density, represented by circles in the figure. The observed pressures at lower densities repre-

\footnotetext{
${ }^{5} \mathrm{H}$. K. Onnes and H. A. Kuypers, Comm. Phys. Lab. Univ. Leiden No. 169a, 16, 5 (1924-26).
}

6 H. M. Kvalnes and V. L. Gaddy, J. Am. Chem. Soc., 53, 394 (1931). 
sented by crosses are lower than the calculated values. This difference appears to be due to a difference between the two sets of experimental data rather than a failure of the equation. Possibly there is a difference in the purity of the two samples.

\section{PROPANE}

The critical volume of propane 0.201 liter per mole was used in place of the value 0.195 chosen by Beattie, Poffenberger, and Hadlock. The value used here corresponds to the mean of the densities of the vapor and of the liquid at $96.70^{\circ} \mathrm{C}$ (about $0.1^{\circ}$ below the critical temperature). The pressures calculated from eq 3 are compared with the values observed for propane in figure 8. Values calculated from the equation agree with the data represented by circles, and agree as well as can be expected with the data for the other sample (represented by crosses), since there is a consistent difference of about 1 part in 1,000 between corresponding pressures for the two samples.

\section{CARBON DIOXIDE}

Two previous papers in which the critical temperature was chosen first ${ }^{7}$ near $31.1^{\circ} \mathrm{C}$ and later ${ }^{8}$ at $30.96^{\circ} \mathrm{C}$ have appeared to be rather contradictory. This apparent contradiction seems to be cleared up

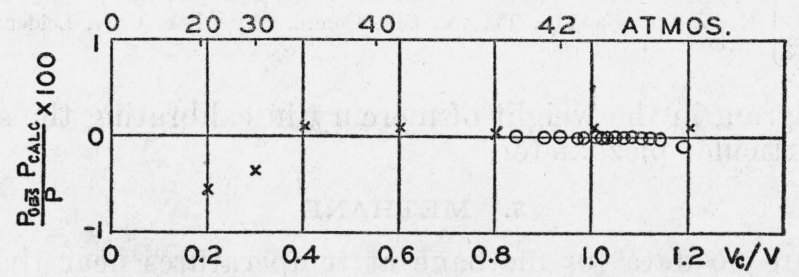

Figure 8.-Comparison of pressures calculated from equation 3 with pressures observed for propane.

$\times$ J. A. Beattie, W. C. Kay, and J. Kaminsky, J. Am. Chem. Soc.'59, ${ }^{r_{1}} 589$ (1937).

O.J. A. Beattie, N. Poffenberger, and C. Hadlock, J. Chem. Phys. 3, 96 (1935).

by the theoretical work of Mayer and Harrison ${ }^{9}$, in which they conclude that a temperature exists for which, at the critical density, $p=p_{c}$, and the first and second derivatives of pressure with respect to volume are zero; but that the sharp dividing surface between the liquid and the gas phases disappears at a slightly lower temperature. For the present purpose, $31.037^{\circ} \mathrm{C}$ was used as the critical temperature, that being the value reported by Michels. The critical density is chosen from Michels' data, but the pressure is that observed at the National Bureau of Standards.

The agreement between the two sets of data (see fig. 9) is remarkable in view of the fact that volumes were only roughly measured in the latter investigation. The calculated pressures agree very well with the observed data, as is shown in figure 9 , the maximum deviation only slightly exceeding 3 parts in 10,000. There is a possibility that these small deviations might be further reduced if the data had been at a temperature about $0.05^{\circ} \mathrm{C}$ higher.

7 C. H. Meyers and M. S. Van Dusen, BS J. Research 10,381 (1933) RP 538.

8 H. T. Kennedy and C. H. Meyers, Refrig. Eng. 15, 125 (1928).

' J. E. Mayer and S. F. Harrison, J. Chem. Phys. 6, 87 (1938). 
The pressures calculated from eq 3 in the range $X=1.1$ to 1.3 are apparently too large. As the density is increased beyond $X=1.3$, the calculated pressures soon become too small and rapidly diverge from the observed values. For example, when $X=1.2$ the pressure observed at this Bureau is $72.97 \mathrm{~atm}$, the calculated 73.13 , or 22 parts in 10,000 higher. For $X=1.3$, the observed pressure is 73.61 atm, whereas the calculated value is 74.04 , or 50 parts in 10,000 higher. Michels reports $81.261 \mathrm{~atm}$ at a density corresponding to

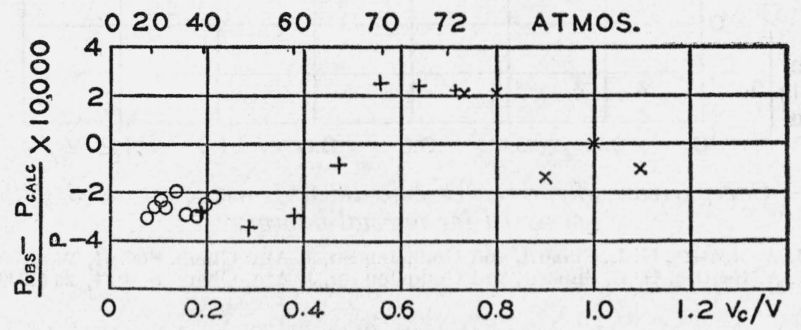

Figure 9.-Comparison of pressures calculated from equation $B$ with pressures observed for carbon dioxide.

$\times$ C. H. Meyers and M. S. Van Dusen, BS J. Research 10, 381 (1933) RP 538.

O+ A. Michels and C. Michels, Proc. Roy. Soc. (London) [A] 153, 201 (1935).

$X=1.485$. Here, however, the calculated value, $80.08 \mathrm{~atm}$, is 161 parts in 10,000 lower. In the region where $X$ is greater than 1.1, the equation deviates in a similar manner in the case of other substances.

\section{PROPYLENE}

The data reported for propylene consist of values of $p V / R T$ tabulated for even reduced pressures. In figure 10 these values are compared with those calculated from eq 3 .

Since the comparison is on the basis of $p V i R T$, a decrease of about 1 percent in the assumed critical volume would be sufficient to cause

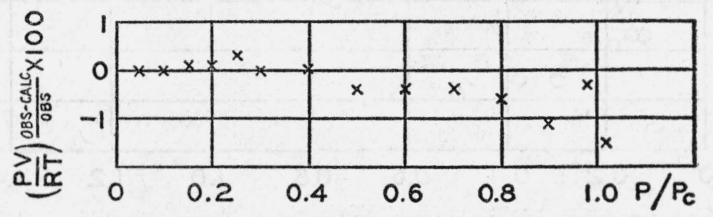

Figure 10.-Comparison of data calculated from equation 3 with data observed for propylene.

W. E. Vaughan and N. R. Graves, Ind. Eng. Chem. 32, 1252 (1940).

practical agreement between calculated and observed values. An accuracy of only 1 percent is estimated by the authors for the data.

\section{NORMAL BUTANE}

The use in this paper of the critical volume 0.2544 liter per mole, together with the other constants in table 2, leads to a value 0.2732 for $p_{c} V_{c} / R T_{c}$. The pressures calculated with this value of the critical volume, together with $n$ from figure 3 , are in slightly better agreement with the observed pressures than those calculated with the aid of the 
reported value, 0.258 liter per mole. The excessive number of decimal places in the value 0.2544 was used for convenience to make the constants in eq 3 the same as for propylene.

As shown in figure 11, the pressures observed near the critical state and the values calculated from eq 3 are in excellent agreement. The difference between the calculated and observed pressures at lower

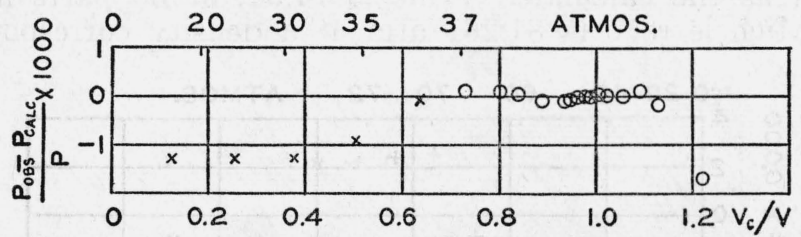

FIGURE 11.-Comparison of pressures calculated from equation 3 with pressures observed for normal butane.

$\times$ J. A. Beattie, G. L. Simard, and Gouq-Jen Su, J. Am. Chem. Soc. 61, 24 (1939).

J. A. Beattie, G. L. Simard, and Gouq-Jen Su, J. Am. Chem. Soc. 61, 26 (1939).

densities amounts to about 1 part in 1,000 , but this difference is only about one-fifth the difference between the observed pressures and values calculated from the equation given by the authors cited in figure 11.

\section{ETHYL ETHER}

As illustrated in figure 12, the difference between the pressures observed for ether and those calculated from eq 3 is less than 2 parts in 1,000 .

10. NORMAL HEPTANE

The comparison of data on heptane in figure 13 suggests a systematic difference between the two sets of observed data. However, this ap-

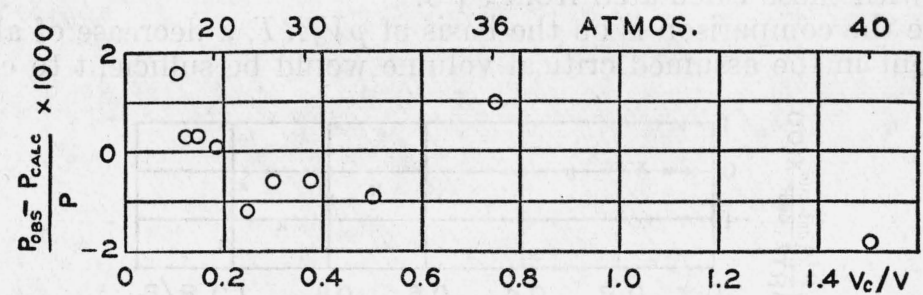

Figure 12.-Comparison of pressures calculated from equation 3 with pressures observed for ethyl ether.

J. A. Beattie, J. Am. Chem. Soc. 46, 342 (1924).

parent difference may be in part caused by errors in extrapolation from $275^{\circ}$ to $267^{\circ} \mathrm{C}$, for the data represented by crosses.

\section{AMMONIA}

The comparison of data for ammonia in figure 14 shows the agreement for low densities (see upper portion of figure) to be excellent not only between the calculated and the observed pressures but also between the two sets of observed data. The deviations from eq 3 rarely exceed 5 parts in 10,000. A study of a graph similar to figure 2, in which other isotherms than the critical are plotted for ammonia, 
gives the impression that this good agreement between the calculated and observed values is somewhat fortuitous. The isotherms at higher temperatures bend upward at low densities too sharply to be represented by eq 3 , whereas the isotherms at lower temperatures are similar to those for water in being convex upward. The calculated

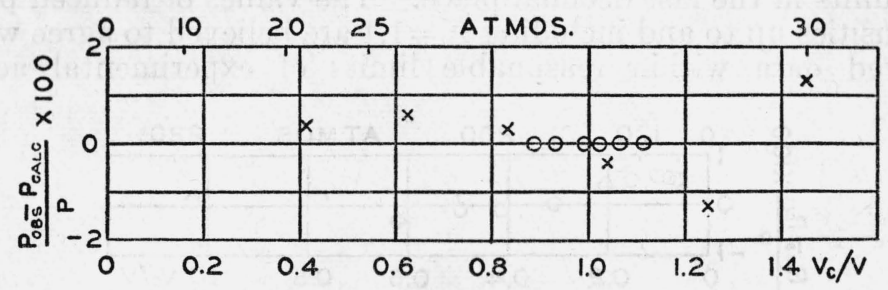

FIGURE 13.-Comparison of pressures calculated from equation 3 with pressures observed for normal heptane.

$\times$ L. B. Smith, J. A. Beattie, and W. C. Kay, J. Am. Chem. Soc. 59, 1587 (1937).

O J. A. Beattie and W. C. Kay, J. Am. Chem. Soc. 59, 1586 (1937).

pressures at higher densities (see lower portion of fig. 14) show larger differences from the observed values; but below the critical density, the differences are probably due to experimental error and uncertainty of extrapolation - in this region there are no corresponding deviations of this size for other substances. The differences at densities greater
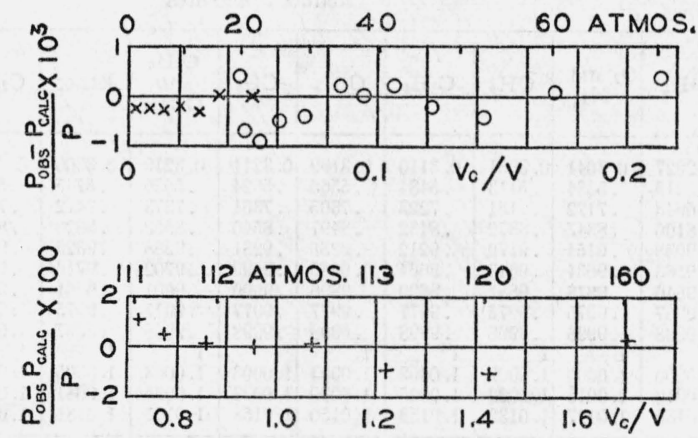

FIGURE 14.-Comparison of pressures calculated from equation 3 with pressures observed for ammonia.

$\times$ C. H. Meyers and R. S. Jessup, Refrig. Eng. 11, 345 (1925).

O J. A. Beattie and C. K. Lawrence, J. Am. Chem. Soc. 52,6 (1930).

+ F. G. Keyes, J. Am. Chem. Soc. 53, 965 (1931).

than the critical are mainly due to inadequacy of eq 3 , as mentioned in the discussion of carbon dioxide.

\section{WATER}

Figure 2 shows that eq 3 does not represent the data for water as satisfactorily as it does the data for other substances. The differences of over $1 / 2$ percent between the observed and calculated pressures, illustrated in figure 15, appear to be greater than can be attributed to experimental error. Water differs from ammonia in that its isotherms when plotted on a graph similar to figure 2 are all convex upward at low densities. 
Table 3 contains values of reduced pressure calculated from eq 3 for 13 substances at various reduced densities, using the constants given in table 2. In some instances the value of the integral in the equation may not have been calculated with sufficient accuracy to determine the corresponding value of the reduced pressure to better than two or three units in the last decimal place. The values of reduced pressure for densities up to and including $X=1.1$ are believed to agree with the observed data within reasonable limits of experimental accuracy

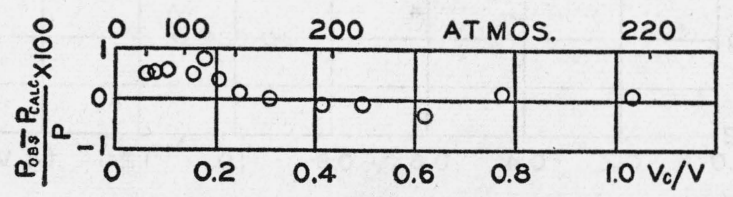

FIGURE 15.-Comparison of pressures calculated from equation 3 with pressures observed for water.

F. G. Keyes, L. B. Smith, and H. T. Gerry, Proc. Am. Acad. 70, 319 (1935).

except in the case of water, where some of the calculated values appear to be in error by about $1 / 2$ percent.

$\mathrm{T}_{\mathrm{ABLE}}$ 3.-Reduced densities and corresponding reduced pressures calculated from equation ( 3 ) for various substances at the critical temperature

\begin{tabular}{|c|c|c|c|c|c|c|c|c|c|c|c|c|}
\hline \multirow[b]{2}{*}{$\boldsymbol{x}$} & \multicolumn{12}{|c|}{ Reduced pressures } \\
\hline & $a \mathrm{H}_{2}$ & ${ }^{6} \mathrm{H}_{2}$ & $\mathrm{O}_{2}$ and & $\mathrm{CH}_{4}$ & $\mathrm{C}_{2} \mathrm{H}_{6}$ & $\mathrm{C}_{3} \mathrm{H}_{8}$ & $\mathrm{CO}_{2}$ & $\begin{array}{l}\mathrm{C}_{3} \mathrm{H}_{6} \\
\text { and } \\
\mathrm{C}_{4} \mathrm{H}_{10}\end{array}$ & Ether & $\mathrm{C}_{7} \mathrm{H}_{16}$ & $\mathrm{NH}_{3}$ & $\mathrm{H}_{2} \mathrm{O}$ \\
\hline $\begin{array}{r}0.1 \\
.2 \\
.3 \\
.4 \\
.5 \\
.6 \\
.7 \\
.8 \\
.9 \\
1.0 \\
1.1 \\
1.2 \\
1.3\end{array}$ & $\begin{array}{r}0.2872 \\
.5132 \\
.6854 \\
.8109 \\
.8992 \\
.9522 \\
.9826 \\
.9960 \\
.9997 \\
1.9999 \\
. .9999 \\
1.0012 \\
1.0141\end{array}$ & $\begin{array}{r}0.2927 \\
.5215 \\
.6943 \\
.8190 \\
.9038 \\
.9563 \\
.9846 \\
.9967 \\
.9998 \\
1 . \\
1.0000 \\
1.0016 \\
1.0130\end{array}$ & $\begin{array}{r}0.3041 \\
.5384 \\
.7122 \\
.8347 \\
.9154 \\
.9634 \\
.9878 \\
.9975 \\
.9998 \\
1 . \\
1.0000 \\
1.0015 \\
1.0127\end{array}$ & $\begin{array}{r}0.3061 \\
.5413 \\
.7151 \\
.8372 \\
.9170 \\
.9643 \\
.9881 \\
.9975 \\
.9998 \\
1 . \\
1.0002 \\
1.0022 \\
1.0132\end{array}$ & $\begin{array}{r}0.3110 \\
.5484 \\
.7223 \\
.8432 \\
.9212 \\
.9664 \\
.9890 \\
.9977 \\
.9998 \\
1 . \\
1.0002 \\
1.0025 \\
1.0138\end{array}$ & $\begin{array}{r}0.3169 \\
.5566 \\
.7305 \\
.8497 \\
.9255 \\
.9685 \\
.9896 \\
.9977 \\
.9998 \\
1.0003 \\
1.0030 \\
1.0150\end{array}$ & $\begin{array}{c}0.3211 \\
.5624 \\
.7361 \\
.8540 \\
.9281 \\
.9699 \\
.9900 \\
.9977 \\
.9998 \\
1 \\
1.0003 \\
1.0033 \\
1.0158\end{array}$ & $\begin{array}{r}0.3219 \\
.5636 \\
.7373 \\
.8550 \\
.9288 \\
.9702 \\
.9901 \\
.9977 \\
.9998 \\
1.00 \\
1.0003 \\
1.0035 \\
1.0161\end{array}$ & $\begin{array}{r}0.3300 \\
.5745 \\
.7472 \\
.8620 \\
.9325 \\
.9716 \\
.9901 \\
.9975 \\
.9997 \\
1.0005 \\
1.0041 \\
1.0181\end{array}$ & $\begin{array}{r}0.3419 \\
.5898 \\
.7606 \\
.8711 \\
.9368 \\
.9737 \\
.9900 \\
.9972 \\
.9996 \\
1 . \\
1.0004 \\
1.0051 \\
1.0210\end{array}$ & $\begin{array}{c}0.3500 \\
.5991 \\
.7670 \\
.8735 \\
.9364 \\
.9708 \\
.9884 \\
.9966 \\
.9995 \\
1 \\
1.0005 \\
1.0062 \\
1.0241\end{array}$ & $\begin{array}{r}0.3689 \\
.6249 \\
.7920 \\
.8935 \\
.9496 \\
.9779 \\
.9914 \\
.9977 \\
.9997 \\
1.0 \\
1.0005 \\
1.0060 \\
1.0241\end{array}$ \\
\hline$\frac{R T_{c}}{p_{c} V_{0}}$ & 3. 203 & 3.273 & 3.422 & 3.448 & 3.513 & 3. 592 & 3. 649 & 3.660 & 3.774 & 3.946 & 4.075 & 4.335 \\
\hline
\end{tabular}

- Calculated with $p_{e}=13.03$ atm, $1 / n=0.060$ (see table 2).

$b$ Calculated with $p_{c}=12.75 \mathrm{~atm}, 1 / n=0.080$ (see table 2).

- Actually calculated as less than unity because of choice of constants (see text).

As already mentioned, two sets of values are given for hydrogen, since the choice of a value for the critical pressure of that substance is to some extent a matter of personal judgment.

The value of $R T_{c} / p_{c} V_{c}$ is also given at the base of the table as an aid in comparing with data for other substances not included in the table. For example, the ratio $R T_{c} / p_{c} \mathrm{~V}_{c}$ for argon is very nearly the same as that for oxygen and nitrogen, consequently the reduced pressures given for those two substances should apply also to argon. It may be convenient also to represent the reduced pressures for other substances by interpolation between the values given in table 3 . 


\section{VIRIAL COEFFICIENTS FOR THE ISOTHERM AT THE CRITICAL TEMPERATURE}

Equation 1 may be transformed to

$$
\begin{gathered}
1-\frac{p V}{R T}=X\left[\frac{3}{2}\left(1-\frac{p_{c} V_{c}}{R T_{c}}\right)+\left\{\frac{3}{2}\left(1-\frac{p_{c} V_{c}}{R T_{c}}\right)-1\right\} \int_{X}^{1} e^{(4 / n)\left(1-X^{n}\right)} d X\right] \\
-\frac{X^{2}}{2}\left(1-\frac{p_{c} V_{c}}{R T_{c}}\right) .
\end{gathered}
$$

Or approximately for small values of $X$

$$
\begin{aligned}
1-\frac{p V}{R T}= & X\left[\frac{3}{2}\left(1-\frac{p_{c} V_{c}}{R T_{c}}\right)+\left\{\frac{3}{2}\left(1-\frac{p_{c} V_{c}}{R T_{c}}\right)-1\right\} \int_{0}^{1} e^{(4 / n)\left(1-X^{n}\right)} d X\right] \\
& -X^{2}\left[\frac{1}{2}\left(1-\frac{p_{c} V_{c}}{R T_{c}}\right)+\left\{\frac{3}{2}\left(1-\frac{p_{c} V_{c}}{R T_{c}}\right)-1\right\} e^{4 / n}\right]
\end{aligned}
$$

The range over which this approximation fulfills a given requirement depends upon the value of $n$. For example, $p V / R T$ calculated from eq 5 does not differ more than 1 in 10,000 from the same quantity calculated from eq 4 for values of $X$ up to 0.5 or 0.6 for $\mathrm{H}_{2}$; up to 0.3 or 0.4 for $\mathrm{CO}_{2}$; and up to 0.1 for $\mathrm{NH}_{3}$.

One may rewrite eq 5 briefly as

$$
p V / R T=1+B X+C X^{2} .
$$

Table 4 gives values of $B$ and $C$ for various substances as calculated from eq 5. The virial coefficients, as sometimes defined from the equation

$$
(p V)_{A}=(R T)_{A}+\frac{B_{A}}{V_{A}}+\frac{B_{A}}{V_{A}^{2}}
$$

can be derived easily from these coefficients.

TABLE 4.-Virial coefficients for critical isotherms

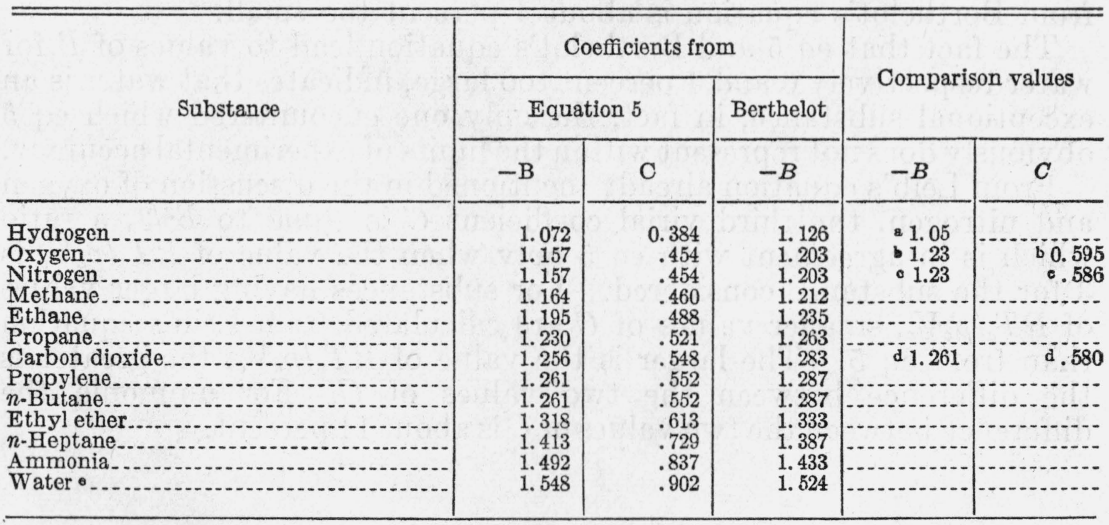

a G. P. Nijhoff and W. H. Keesom, Comm. Phys. Lab. Univ. Leiden No. 188 e 17 (1924-28).

b H. A. Kuypers, Comm. Phys. Lab. Univ. Leiden No. 169b, 16 (1924-26).

- A. Th. van Urk, Comm. Phys. Lab. Univ. Leiden No. 169e, 16 (1924-26).

d Proc. Roy. Soc. (London) [A] 153, 201 (1935).

- Allowing for the effect of $C$, the value of $B$ estimated from the 2 lowest observed pressures is about 1.46. 
The fourth column of table 4 contains values of $-B$ calculated from Berthelot's equation

$$
p V=R T\left[1+\frac{9 p T_{c}}{128 p_{c} T}\left(1-6 \frac{T_{c}{ }^{2}}{T^{2}}\right)\right],
$$

which leads to a value $-45 R T_{c} / 128 p_{c} V_{c}$ for $B$ at the critical temperature. Comparison values presented by other authors are given in some cases.

In general, the agreement of pressures calculated from eq 3 has been shown to be very good, hence the values of $B$ in table 4 obtained from eq 5, with the exception of that for water, are considered to be within 2 percent or less of the correct values.

The accuracy of the data on hydrogen is not a sufficient basis for a choice between the coefficient calculated from eq 5 and that given by the Leiden Laboratory.

The data on oxygen and nitrogen confirm the coefficients presented in table 4 from eq 5 . The value 1.23 given by the Leiden Laboratory for $B$ for oxygen and nitrogen, in other words, the intercept for the critical isotherm at zero density in figures 5 and 6 is out of line with the experimental data for both substances.

The discrepancy of about $1 / 2$ percent between the value of $B$ for $\mathrm{CO}_{2}$ calculated from eq 5 and that given by Michels, is insignificant for the purpose of calculating pressures at low densities. For example, for $1 \mathrm{~atm}$ eq 5 gives $p V \mid R T=0.99526$, whereas Michel's equation gives 0.99524 ; even at $19 \mathrm{~atm}$ the difference in $p V / R T$ amounts to only 3 parts in 10,000 .

It will be seen from table 4 that the values of $B$ from Berthelot's equation are about 5 percent too high at the top of the table, but for larger values of $R T_{c} / p_{c} V_{c}$ (see table 2), the difference between the two sets of values decreases and changes sign between ethyl ether and $n$-heptane.

The deviations shown in figure 15 indicate that pressures calculated from eq 3 are either in agreement with or slightly greater than the observed data for ammonia at the lowest densities. Consequently, the value of $B$ from eq 5 in table 4 is not too large, and the value of $B$ from Berthelot's equation is about 4 percent too small.

The fact that eq 5 and Berthelot's equation lead to values of $B$ for water respectively 6 and 4 percent too large, indicates that water is an exceptional substance, in fact, the only one encountered which eq 5 obviously does not represent within the limits of experimental accuracy.

From Leib's equation already mentioned in the discussion of oxygen and nitrogen, the third virial coefficient $C$ is equal to $B^{2} / 3$, a ratio which is in agreement with eq 5 only when the value of $R T_{c} / p_{c} V_{c}$ is 3 for the substance considered. For substances having larger values of $R T_{c} / p_{c} V_{c}$, smaller values of $C$ are calculated from Leib's equation than from eq 5. The larger is the value of $R T_{c} / p_{c} V_{c}$, the greater is the difference between the two values of $C$. For ammonia the difference between the two values of $C$ is about 11 percent. 


\section{FUGACITY ALONG THE ISOTHERM AT THE CRITICAL TEMPERATURE}

For the purpose of calculating fugacity along the critical isotherm, an expansion of the integral appearing in eq 3 into series form is convenient. Then solving for $\partial\left(p / p_{c}\right) / \partial X$ by differentiating that equation, substituting this derivative in the equation

$\ln \frac{f}{p}=-\frac{1}{R T_{c}} \int_{0}^{p} \alpha d p=\frac{1}{R T_{c}} \int_{0}^{p} V d p-\int_{0}^{p} \frac{d p}{p}=\frac{p_{c} V_{c}}{R T_{c}} \int_{0}^{x} \frac{1}{X}\left(\frac{\partial p / p_{c}}{\partial X}\right) d X-\int_{0}^{p} \frac{d p}{p}$

and integrating, one obtains

$$
\begin{gathered}
\ln \frac{f}{p}=\ln \frac{R T_{c}}{p V}-3\left(1-\frac{p_{c} V_{c}}{R T_{c}}\right)\left(X-\frac{X^{2}}{4}\right) \\
-\left(1-\frac{3 p_{c} V_{c}}{R T_{c}}\right) e^{4 / n}\left\{1-\frac{4 / n}{n+1}+\ldots+\frac{(-1)^{m}(4 / n)^{n}}{(m n+1)(m !)}+\ldots\right\} X \\
+\frac{1}{2}\left(1-\frac{3 p_{c} V_{c}}{R T_{c}}\right) e^{(4 / n)}\left\{\frac{3}{2} X^{2}-\frac{(4 / n)(n+3) X^{n+2}}{(n+1)(n+2)}+\ldots\right. \\
\left.+\frac{(1-)^{m}(4 / n)^{m}(m n+3) X^{m n+2}}{(m n+1)(m n+2)(m !)}+\ldots\right\}
\end{gathered}
$$

For a hypothetical substance with a value of 3 for $R T_{c} / p_{c} V_{c}$, eq 7 becomes

$$
\ln \frac{f}{p}=\ln \frac{R T_{c}}{p V}-3\left(1-\frac{p_{c} V_{c}}{R T_{c}}\right)\left(X-\frac{X^{2}}{4}\right)
$$

and for the critical state of such a substance, $\ln \left(f_{c} / p_{c}\right)=(\ln 3)-1.5$, or $f_{c} / p_{c}=0.6694$. For carbon dioxide with the constants in table 2, $c / p_{c}=0.6593$. This is in perfect agreement with the value interpolated between the data given by Deming and Deming. ${ }^{10}$

For carbon dioxide under a pressure of $1 \mathrm{~atm}$, the equation presented in this paper leads to $p V / R T=0.99526$ and to $f / p=0.99528$, whereas the corresponding value given by Deming and Deming for $f / p$ is 0.99504 . The fact that Michels obtains $p V / R T=0.99524$ and that $\mathrm{f} / p$ should approximate $p V / R T$ very closely, confirms the value 0.99528 or $f / p$ for carbon dioxide at $1 \mathrm{~atm}$ and the critical temperature.

It is to be noted that the value of $f / p$ at the critical state is very slightly over $2 / 3$ when $R T_{c} / p_{c} V_{c}=3$ and decreases only slightly with increase in $R T_{c} / p_{c} V_{c}$, so that for any substance at the critical state, $f / p$ deviates from $2 / 3$ by only a few percent.

The equation

\section{SUMMARY}

$$
\frac{V\left(R T_{c}-p V\right)}{V_{c}\left(R T_{c}-p_{c} V_{c}\right)}=\frac{3}{2}-\frac{\dot{i}}{2} X+\left(\frac{3}{2}-\frac{1}{1-p_{c} V_{c} / R T_{c}}\right) \int_{X}^{1} e^{(4 / n)\left(1-X^{n}\right)} d X,
$$

where $n$ is an empirical constant and $X=V_{c} / V$, the reduced density, has been shown to fulfill the requirements at the critical state and to

\footnotetext{
${ }^{10}$ W. E. Deming and L. S. Deming, Phys. Rev. 56, 108 (1939).
} $469958-42-5$ 
represent the critical isotherms for 12 out of 13 substances in the range of densities up to 1.1 times the critical density, within the reasonable limits of experimental error. The deviations for the one exception, water, amount to $1 / 2$ percent, which exceeds the tolerance set by the experimental accuracy. For the very accurately measured critical isotherm of carbon dioxide, the differences between calculated and observed pressures do not exceed 3 parts in 10,000.

Of the five constants in the equation, one is limited to a single value for a simple solution, three are directly determined by the critical data, which when substituted in the equation make it automatically fulfill the requirement of the critical state, that the first and second derivatives of pressure with respect to density shall be zero. The reciprocal of the fifth constant, $n$, has been shown to be very nearly a linear function of $R T_{c} / p_{c} V_{c}$. In fact, when $R T_{c} / p_{c} V_{c}$ does not exceed 3.8 or 3.9 , this linear relation established by the values determined for hydrogen and for carbon dioxide furnishes a correlation from which $n$ may be determined for other substances, and consequently, the critical isotherm may be predicted with great accuracy for densities up to 1.1 times the critical density. The value of $n$ for substances with larger values of $R T_{c} / p_{c} V_{c}$ will probably need to be determined individually, although even then pressures may be predicted within a few percent, from the critical data and the linear relation mentioned for the fifth constant.

For low pressures the equation may be reduced to one expressing $p V / R T$ as a quadratic function of the density, from which pressures may be calculated with an accuracy exceeding that of most measurements.

Although the equation is applied only to the critical temperature, the wide range of densities covered with such accuracy makes it valuable for establishing the critical isotherm as a base line in the tabulation of smoothed values of the properties of substances.

Values of fugacity along the critical isotherm can be calculated from the equation. The fugacity at the critical state is very nearly twothirds the critical pressure for all substances, but varies slightly with the value of $R T_{c} / p_{c} V_{c}$.

The author acknowledges with thanks several suggestions from Carl S. Cragoe for the improvement of this paper.

WASHington, February 11, 1942. 